\title{
Report Card on Katrina: Administrative Progress Four Years Hence
}

\author{
Carole L. Jurkiewicz
}

Published online: 24 September 2009

(C) Springer Science + Business Media, LLC 2009

With the benefit of four years' distance from the initial shock of the disaster itself, and the emotional outrage of administrative failures in the wake of Katrina, we can now address the efficacy of both recovery programs and leadership efforts with greater objectivity. Ranked as the second worst natural disaster in U.S. history and one of the fifteen worst globally (LiveScience 2009), heightened attention is paid to the lessons learned from post-Katrina recovery efforts, with an eye toward what can be done to contain and mitigate damage resultant of future such events. With the U. $\mathrm{S}$. heretofore positioned as the most successful industrialized nation in the world, the spotlight is on how well we have applied our resources, knowledge, technology, management and communication skills, and governmental and social networks to redress the consequences of Katrina on our citizenry, economics, and business sectors. From the key top scholars who contributed to a volume focusing on administrative failure in the immediate wake of Hurricane Katrina (Jurkiewicz 2007), those whose work included measurables that could be realistically reassessed within the four year period hence were invited to revisit their respective areas of study. They were asked to re-examine these aspects of administrative failure to assess progress as well as to analyze what, if progress has not occurred, were the factors inhibiting recovery. Their analysis and insights bring to light many dimensions of recovery that will be useful not only in furthering efforts to rectify the damage of this particular disaster, but for all those interested in emergency and

C. L. Jurkiewicz $(\bowtie)$

Louisiana State University, Baton Rouge, LA, USA

e-mail: cljrkwcz@1su.edu 
disaster management and recovery worldwide. As is revealed through these series of articles, if hindsight is $20 / 20$, the road ahead is long and replete with hurdles. Not only in resource allocation and infrastructure restoration can we identify clear needs for advancement, but what has become increasingly obvious is that the roadblocks to progress are more often political and cultural rather than resource-based. The implications for future recovery efforts, as well as for those still directed at the effects of Katrina, are a need to extend mitigation efforts beyond resource delivery and management to include political, cultural, and social intervention. The authors offer fresh perspectives on progress post-Katrina, concise analysis of what has been effective and what needs to be done, and suggestions on where we need to focus our efforts into the future.

Ivor van Heerden, as Director and Lead Principal Investigator of the LSU Hurricane Center and the head of the Louisiana's forensic levee investigation postKatrina, along with his co-authors, begin this issue with a key report on what factors contributed most to the devastating floods following the impact of the Hurricane. "How a Navigation Channel Contributed to Most of the Flooding of New Orleans During Hurricane Katrina" brings to bear the top researchers, instrumentation, and investigative skills yet applied in forensic disaster analysis. Their report reveals how the design and lack of maintenance facilitated the flooding of over $85 \%$ of New Orleans and, even more alarmingly, what impact the recent Hurricane Gustav foretells about the likelihood of these levees to fail again in future storms if steps are not taken to correct the problems. Louise Comfort and her co-authors further this progress assessment in their article, "The Dynamics of Disaster Recovery: Resilience and Entropy in Hurricane Response Systems, 2005-2008". The need for both awareness and proactive attempts to balance the dynamics of resilience (the catalystic effect of the disaster to expend energy and make progress), and entropy (when the initial motivation subsidies and ennui ensues), is essential in sustaining risk reduction and recovery efforts. The empirical approach they use to measure the presence and extent of resilience and entropy is novel and enlightening, and likely to set a standard for such research into the future. To go beyond making the logical argument, which they do well, and triangulate data from multiple time points allows the reader to draw profound conclusions and practical suggestions for emergency and disaster management. Beverly Cigler's article, "Post-Katrina Hazard Mitigation on the Gulf Coast", demonstrates the effectiveness of comparison-modeling in assessing the risks and potential effectiveness of mitigation efforts. Her review of both Hurricanes Katrina and Ike, which share many similarities, concludes with clear recommendations for policy development and explores options for reducing exposure and minimizing adverse consequences. Ultimately, her study calls for comprehensive and holistic changes in the management of the Mississippi River system as well as policy change and insurance reform. This insightful retrospective of what has been learned articulates an urgent need for effective, informed, objective leadership. Whereas most analysis in the popular media focuses upon the lack of financial resources, Cigler makes a cogent argument for the necessity of both material resources and effective management skills in emergency/disaster response and mitigation.

William Waugh focuses the reader's attention on the leadership factor in assessing post-Katrina recovery efforts. "Katrina and the Governors" reviews and compares 
the actions, policies, and political acumen of Louisiana's and Mississippi's governors and what their efforts have contributed to the progress or hindrance of disaster mitigation in the region. He examines why the programs in both states have been slow and contentious, and questions whether these efforts offer any promise for reducing risks in future disasters. As is clear, one can see what the right thing to do is, but getting the parties to act rationally in the process is something altogether different. Can policies and programs triumph over diffusion of responsibility and political agendas? It's the ongoing challenge of government that comes into acute focus when viewed through the lens of post-Katrina recovery. In "The Effects of Political Leadership and Cultural Ethics on Recovery in Post-Katrina Louisiana" this author discusses how the renowned style of politics and cultural elements of Louisiana stand as the greatest obstacle to recovery post-Katrina. While acknowledging the failures of resource management, communication, engineering, and policy, this article zeros in on the ethical factors that serve as both cause and effect in Louisiana politics and culture, and why nothing short of a cultural revolution will advance the hopes for effective recovery and redevelopment. The lessons learned from this predicament offer insight beyond Louisiana to the impact of cultural influences on rational behavioral models, and the inherent struggle between selfinterest and the greater good in governance. Gloria Simo provides a prescriptive advise for collaboration in her article, "Sustaining Cross-Sector Collaborations: Lessons from New Orleans". Little has been written in this key area of disaster recovery and Simo documents not only best collaborative practices, but also how to sustain such relationships and efforts well beyond the initial emergency. As the dynamics and requirements of the situation shift over time, as new needs emerge and public/private sector resources and foci change, collaborative relationships need to be both stable and malleable if they are to be sustained and effective. Simo offers much needed insight into the particulars of how this works, and why.

James Garnett and Alex Kouzmin provide an update and refreshing analysis of the role of communication in disaster mitigation in their article, "Crisis Communication Post Katrina: What Are We Learning?" Their vulnerability audit of communication capabilities during crises points to the influence of political and administrative changes on the effectiveness of message transmission. In their original work (see Jurkiewicz 2007), they identified an over-dependence on interpersonal influence, media relations, and showcasing technologies as impediments to effective crisis communication. They extend this analysis of capacity and dependency issues and identify the need for significant changes in government policy to avoid the exploitation and privatization of disasters they contend occurred in the immediate wake of Katrina. Ali Farazmand's article, "Hurricane Katrina, the Crisis of Leadership, and Chaos Management: Time for Trying the Surprise Management Theory" completes this special issue with a global perspective on the U.S.'s lack of effectiveness in disaster mitigation. Elucidating how the world views our response as an overwhelming failure of governance, leadership, and public management and calling into question our system's legitimacy to deal with such issues, he suggests a solution. Surprise Management Theory is the most effective method of chaos management, he argues. From the ruins of Katrina he sees hope in the form of a new approach to responsive and effective crisis and disaster management. 
At the conclusion of reading this set of articles on administrative progress postKatrina, a common theme emerges. Our collective knowledge of what works best in both planning for and mitigating the consequences of disasters on the scale of Katrina, has grown exponentially and led to some very clear directives for both policy and practice. Such solutions inform crisis, emergency, and disaster management at scales both small and large, and toward that end such research has proven invaluable. Yet the biggest hurdle to implementation of these best practices appears to be the human factor. Politics, culture, ethics, self-interest, and protection of one's scope of influence above rational choice is the next and arguably more difficult challenge in advancing the effectiveness of disaster management. Perhaps the work of the scholars presented here, along with others, will lead to clearer avenues of progress when we view Katrina a decade hence.

\section{References}

Jurkiewicz, C.L., Ed. 2007. Administrative Failure in the Wake of Katrina. Special Issue: Public Administration Review, 67(6)

LiveScience (2009). http://www.livescience.com/environment/top-10-natural-disasters-1.html

Carole L. Jurkiewicz Ph.D. is the Woman's Hospital Distinguished Professor of Healthcare Management and the John W. Dupuy Endowed Professor in the Public Administration Institute of the E.J. Ourso College of Business at Louisiana State University. Her work focuses upon organizational performance as a function of employee ethicality and value identification. She has published widely in the areas organizational and individual performance, ethics, power, and leadership, bringing to her academic career many years experience as an executive in private and nonprofit organizations. She has served as a consultant to many organizations including IBM, USPS, National Public Radio, American Marketing Association, and many governmental departments at the federal, state, and local levels. 